Case Report

\title{
DOUBLE SUPERIORVENACAVA AND ITS ASSOCIATED CLINICAL IM PLICATIONS - A CASE REPORT AND LITERATURE REVIEW
}

\author{
Sushma R Kotian ${ }^{1}$, Antony Sylvan D Souza ${ }^{2}$, Praveena Ravichandran ${ }^{3}$, Pallavi Bhat ${ }^{4}$ \\ \& Mamatha Hosapatna ${ }^{4}$ \\ Lecturer ${ }^{1}$, Professor $\&$ HOD $^{2}$, Postgraduate ${ }^{3}$, Associate Professor ${ }^{4}$, Department of Anatomy, Kasturba M edical College, \\ M anipal University, Manipal, Karnataka, India. \\ Correspondence : \\ Mamatha $\mathbf{H}$ \\ Associate Professor, Department of Anatomy, Kasturba M edical Collage \\ Manipal University, M anipal 576104, Karnataka, India \\ Mobile : +91 8202922327 E-mail : mamatha2010@yahoo.com
}

\begin{abstract}
:
Abnormalities of the vascular system are always of extreme interest due to its importance in circulation. Normally the superior vena cava is a single vascular structure formed by the union of right and left brachiocephalic veins which are in turn formed by the union of corresponding internal jugular and subclavian veins, draining the head and neck as well as the superior extremity. However during routine dissection in the Department of Anatomy, Kasturba M edical College, Manipal, we came across a case of double superior vena cava with persistent left superior vena cava in a 58-year-old male cadaver. Both the vena cavae were formed as continuations of brachiocephalic veins of the corresponding side. The persistent left superior vena cava opened into the enlarged coronary sinus that drained into the right atrium between the opening of inferior venacava and right atrioventricular orifice. No communication was observed between the two vena cavae. A persistent left superior vena cava does not by itself produce any physiological derangement. But it has important clinical implications in certain clinical interventions. It may complicate placement of cardiac catheters or pacemaker leads. Awareness of this anomaly may therefore reduce confusions and thus would help to avoid further complications.
\end{abstract}

Keywords : persistent left superior vena cava, coronary sinus, superior vena cava, right atrium

\section{Introduction:}

Abnormalities in the cardio-vascular system are of extreme interest because of their significance in different developmental problems and their effect on the organ of circulation.

Normal anatomy describes the formation of a single superior venacava by the union of right and left brachiocephalic veins which are in turn is formed by the union of corresponding internal jugular and subclavian veins, draining the head and neck as well as the superior extremity.

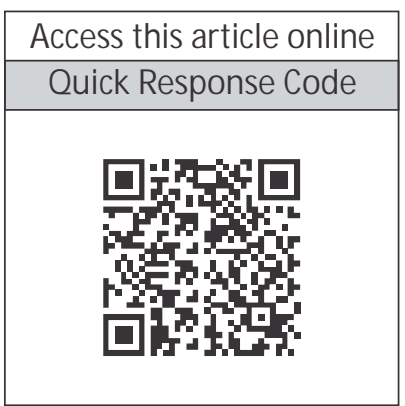

Double superior vena cava (SVC) with the persistent left superior vena cava (PLSVC) is a rare venous malformation. Patients with PLSVC may have other associated cardiac malformations such as atrial septal defect, ventricular septal defect or endocardial cushion defect. ${ }^{1,2}$ Presence of PLSVC may also interfere and cause problems during various invasive procedures such as pacemaker implantation, central venous catheterisation, retrograde delivery of cardioplegia and retrograde left ventricular pacing. ${ }^{3.5}$ The present case reports the existence of one such anomalous PLSVC in an adult cadaver.

\section{Case Report :}

During routine dissection in the department of Anatomy, Kasturba M edical College, M anipal, we came across a case of double superior vena cava with persistent left superior vena cava (PLSVC) in a 65-year-old male cadaver. Both the vena cavae were formed as continuations of brachiocephalic veins of the corresponding sides. The PLSVChad the same length and caliber compared to the superior vena cava (Figure 1). When traced, it opened into the enlarged coronary sinus that further drained into the right atrium between the opening of inferior venacava and 
right atrioventricular orifice (Figure 2).There was no communication between the two vena cavae (Figure 1). The hemiazygos and accessory hemiazygos veins drained normally into the azygos vein which in turn drained into the right superior vena cava. No other associated variations were observed.

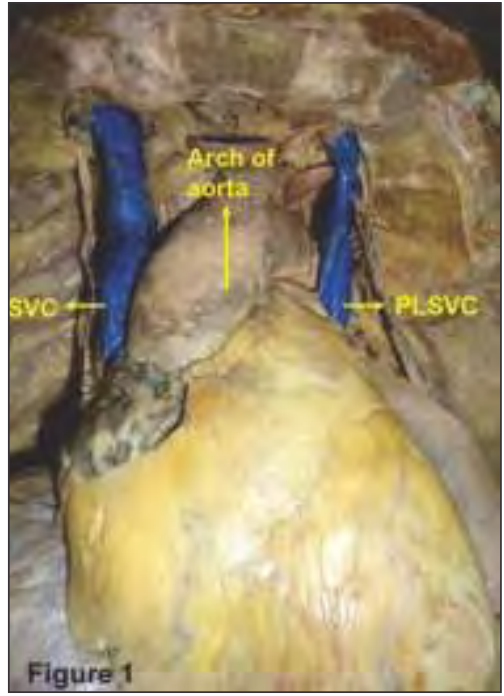

Figure 1: Showing persistent left superior vena cava (PLSVC). Both the vena cavae (SVC \& PLSVC) were formed as continuations of brachiocephalic veins of the corresponding side. No communication was observed between the two veins. SVC: Superior vena cava, RA: Right auricle.

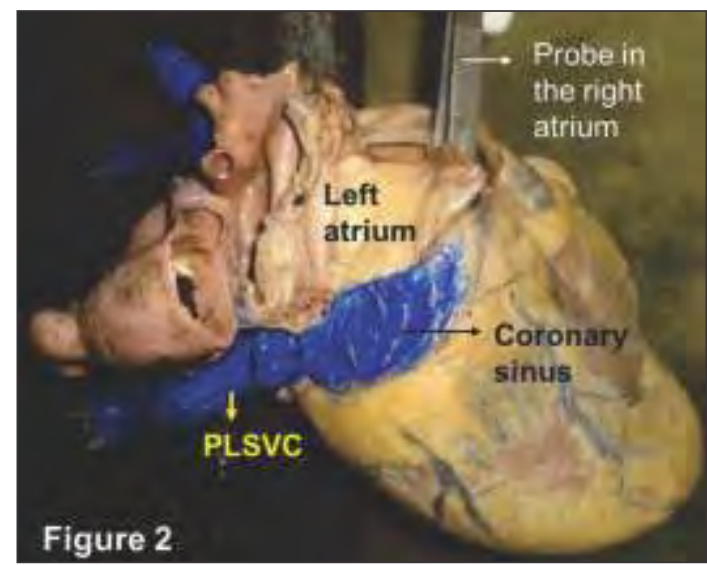

Figure 2: Showing the persistent left superior vena cava (PLSVC) opening into the enlarged coronary sinus that drains into the right atrium between the opening of inferior vena cava and right atrioventricular orifice.

\section{Discussion:}

Double superior vena cava with a PLSVC is a rare anomaly. It is estimated to exist in $0.3-0.5 \%$ of the general population and $3-10 \%$ of patients with other forms of congenital heart disease. It is a remnant of a vessel which persists as an embryological counterpart of the normal right-sided superior vena cava. ${ }^{6}$

During the fifth week of intrauterine life, in the human fetus, three pairs of major veins can be distinguished: the vitelline veins, carrying blood from the yolk sac to the sinus venosus; the umbilical veins, originating in the chorionic villi and carrying oxygenated blood to the embryo; and the cardinal veins, draining the body of the embryo proper. The cardinal veins form the main venous drainage system of the embryo. This system consists of the anterior cardinal veins, which drain the cephalic part of the embryo, and the posterior cardinal veins, draining the remaining part of the body of the embryo. The anterior and posterior veins join to form common cardinal veins and enter the right and left horns of the sinus venosus. Formation of the vena cava system is characterized by the appearance of anastomoses between the left and right sides in such a manner that the blood from the left side is directed to the right side. The anastomosis between the anterior cardinal veins develops into the left brachiocephalic vein. M ost of the blood from the left side of the head and the left upper extremity is thus directed to the right. The terminal portion of the left anterior cardinal vein entering into the left brachiocephalic vein is retained as the left superior intercostal vein. The superior vena cava is thus formed by the right common cardinal vein and the proximal portion of the right anterior cardinal vein. On the other hand, the left common cardinal vein and the distal part of the left horn become atretic and forms the ligament of Marshall or ligament of the left superior vena cava. If this normal regression of the left cardinal vein fails to occur, it results in a PLSVC. ${ }^{7}$

Reports have stated the variations and abnormalities related to double superior vena cava. ${ }^{1-4,8}$ The most common thoracic venous abnormality is the LPSVC draining into the coronary sinus in the presence of both left and right superior vena cavae. This anomaly is usually asymptomatic and does not require treatment unless accompanied by other cardiac anomalies. ${ }^{9 \cdot 11} \mathrm{~A}$ bridging innominate vein is usually observed in these cases. ${ }^{8}$ 
However, in the present case, a double SVC with a PLSVC was observed and there was no communication between the two superior vena cavae unlike as reported previously.

Rarely, the left SVC may also drain into the left atrium as a result of arterial desaturation. ${ }^{11}$

This anomaly was observed in approximately $7.5 \%$ of cases of LPSVC, and it results in a small right to left shunt. This has a minor haemodynamic effect, mainly a variable degree of systemic cyanosisand may lead to clinical symptoms. ${ }^{1,8}$

The left SVC has also been reported to be identifiable in the fetus and be accompanied by coarctation and arch hypoplasia. ${ }^{12}$

PLSVC may also give rise to rhythm disturbances such as sinus node dysfunction and atrioventricular block. These rhythm problems may be related to the stretching of the conduction tissue caused by the enlargement of the coronary sinus. ${ }^{1,4,5}$ It may also be associated with other malformations such as situsinversus or tetralogy of Fallot. ${ }^{7}$ Detailed and accurate echocardiographic studies is therefore useful in identifying this rare congenital defect, thus avoiding further complications during invasive procedures such as cardiac pacemaker implantation, resynchronization therapy, radiofrequency catheter ablation, internal jugular or subclavian vein catheter insertion. ${ }^{1-4}$

During cardiac surgery, the presence of PLSVC would be a relative contraindication to the administration of retrograde cardioplegia. It may be possible to clamp the PLSVC to avoid the cardioplegia solution from perfusing retrograde up the PLSVC and its tributaries. However, there is a possibility that there may be some steal of cardioplegia solution through an accessory vein. Further, the coronary sinus catheter balloon may not be able to occlude the dilated coronary sinus. This may result in the failure of flow of cardioplegia solution to the myocardium. Thus, the cardioplegia solution administered would largely be distributed to the left internal jugular and left subclavian veins, rather than the myocardium. ${ }^{8}$

If the right superior vena cava is absent, all venous return from the upper body will drain through the LPSVC. Hence, this may affect the use of the retrograde cardioplegia. In such a case, occlusion or ligation of the LPSVC would be fatal as this may result in cerebral congestion. ${ }^{6}$

During heart transplantation in a patient with PLSVC, the coronary sinus must be dissected carefully to permit reanastomosis of PLSVC to right atrium. ${ }^{5,6}$ Therefore, both surgeons and perfusionists should be aware of the anatomy of PLSVC and its associated intraoperative complications.

CT or M RI is an important non-invasive diagnostic tool for accurate diagnosis of this rare congenital venous malformation. ${ }^{6}$ The left SVC opening to the right atrium through the coronary sinus can be clearly shown by MPR and 3D VR obtained through M DCT as indicated by Onbas etal. ${ }^{13}$

Double SVC is a rare congenital anomaly and is sparsely available in the medical literature. Therefore both clinicians and sonographers should be alerted about the possible existence of this venous anomaly, other cardiac abnormalities associated with it and their clinical consequences so as to prevent possible complications in routine clinical practice and during cardiopulmonary bypass. The present case report adds to the existing knowledge of these congenital abnormalities and stresses on the use of different diagnostic techniques for its accurate diagnosis thereby avoiding further complications while planning different interventions.

\section{Acknowledgements:}

We are grateful to the cadaver donors who have voluntarily donated the same for the purpose of medical research. 


\section{References:}

1. Erdogan M, Karakas P, Uygur F. Persistent left superior vena cava: the anatomical and surgical importance. West Indian M ed J. 2007; 56: 72 76.

2. Ying $Z Q, M a J, X u$ G, Chen $M Y$. Double superior vena cava with a persistent left superior vena cava. Intern M ed. 2008; 47: 679-680.

3. Biffi M, Boriani G, Frabetti L, Bronzetti G, BranziA . Left superior vena cava persistence in patients undergoing pacemaker or cardioverterdefibrillator implantation: a 10-year experience. Chest. 2001; 120 : 139-144.

4. Chandra A, Reul GJ Jr. Persistent left superior vena cava. Discovered during placement of central venous catheter. Tex Heart Inst J. 1998; 25: 90.

5. Goyal SK, Punnam SR, Verma G, Ruberg FL. Persistent left superior vena cava: a case report and review of literature. Cardiovasc Ultrasound. 2008; 6: 50.

6. Yurtdas M, Sahin M.Double superior vena cava (persistent left superior vena cava draining into the coronary sinus) - case report.Eastern Journal of M edicine. 2013; 18: 23-25.
7. Sadler TW.Langman's Medical Embryology. 7th ed. Baltimore: Williams and Wilkins. 1995; 221-3.

8. Paval J, Nayak S. A persistent left superior vena cava. Singapore M ed J. 2007;48: 90-93.

9. Gerber TC, Kuzo RS. Images in cardiovascular medicine. Persistent left superior vena cava demonstrated with multislice spiral computed tomography. Circulation. 2002; 105:79

10. Minniti S, Visentini S, Procacci C. Congenital anomalies of the venae cavae: embryological origin, imaging features and report of three new variants. EurRadiol. 2002; 12:2040-2055

11. Park MK. Pediatric cardiology for practitioners. 4th ed. St. Louis: Mosby. 2002; 141- 263.

12. Pasquini L, Fichera A, Tan T, Ho SY, Gardiner H. Left superior caval vein a powerful indicator of fetal coarctation. Heart. 2005; 91:539-540.

13. Onbas O, Kantarci M, Koplay M, Olgun H, Alper F, Aydinli B, Zirek H, CevizN. Congenital anomalies of the aorta and vena cava: 16-detectorrow CT imaging findings. DiagnIntervRadiol. 2008; 13:163-171 